\title{
Overview of therapeutic options in COVID-19
}

\author{
Sarthak Katyal $^{1}$, Dr. Swarupa Chakole ${ }^{2}$ \\ ${ }^{1}$ Medical Intern, Jawaharlal Nehru Medical College ,Datta Meghe Institute of Medical Sciences(Deemed to be \\ University), Wardha(442001)Maharashtra, India \\ ${ }^{2}$ Professor, Dept. of Community Medicine, Jawaharlal Nehru Medical College, Datta Meghe Institute of \\ Medical Sciences (Deemed to be University), Wardha(442001) Maharashtra, India \\ Email:sarthakkatyal91@gmail.com
}

\section{Corresponding author:}

SarthakKatyal

Medical Intern, Jawaharlal Nehru Medical College, Datta Meghe Institute of Medical Sciences (Deemed to be University), Wardha(442001) Maharashtra, India

Review Article

Conflict of Interest: None

\begin{abstract}
:
Coronavirus is essentially a respiratory sickness brought about by a newfound rSARS-CoV-2 infection and distinguished in the city of Wuhan, China in December 2019. The emerging outbreak of Covid disease 2019 (COVID-19) brought about because ofthe severe respiratory disorder Covid 2 (SARS-CoV-2) presents a phenomenal test for medical services frameworks around the world.WHO has proclaimed this illness as a pandemic, and cautioned different nations. Like other Covids, this may create respiratory plot contaminations in the patients range from gentle to lethal ailment like pneumonia and ARDS(acute respiratory distress syndrome). The features of coronavirus and the capacity to quickly make far reaching contamination has significant ramifications, justifying vivacious disease avoidance and the preventive measures. While the affirmed quantity of the cases have outperformed 10.3 million throughout the world and keeps on developing, as the possible seriousness related to infection along with its destructive confusions needs critical advancement of the novel restorative specialists to both forestall and cure the COVID-19 illness . In spite of the fact that antibodies and explicit medication treatments presently can't seem to be found, progressing investigation and subjective preliminaries have led to the examination of viability of the reused medications for curing COVID19 illness .According to the current audit, some of the medication competitors have been recommended to cure COVID-19 will be talked about. While these incorporate enemy of the viral specialists (remdesivir, rebetol, lopinavir-ritonavir,choloroquine, favipiravir, hydroxychloroquine, umifenovir ,oseltamivir,), immunomodulating based specialists (interferons, plasma bondings , tocilizumab), (azithromycin, corticosteroids), along with other random specialists. With components of activity and further pharmacology based property which should be investigated, within a specific spotlight on the proof base wellbeing with viability of a every specialist.
\end{abstract}

Keywords: Coronavirus, COVID-19 illness, Medication targets, subjective preliminaries, Procedure of activity

\section{INTRODUCTION :}

Reusing old medications attempting to treat new illnesses, safeguarding racked sedates and broadening licenses' lives can make drug for repurposing (otherwise called as repositioning of drug) ,alluring type for medication disclosure [1]. Repurposing can assist with recognizing new treatments for infections at a lesser cost and within more limited time period, especially in situations when preclinical security contemplates had just finished [2].
It could assume a vital part for "remedial delineation method" in patient with uncommon, unpredictable ,ongoing illnesses with less powerful or no advertised therapy alternatives accessible [3]. Until this point, the most striking repurposed drugs have been found either through luck, in view of explicit pharmacological experiences or utilizing test screening stages [4].The coming of genome related innovations and calculational methodologies had prompted the advancement of new methodologies for 
drug related repositioning. Along with medication relatable information development along with information activities that are open, bunch of different repositioning procedures along with methods had arisen along with coordinating information among different sources like pharmacology based, hereditary, compound or clinic based information. This strategy could collect proof supporting disclosure of different uses or signs for already existing medications [5]. About the viability related to this methodology it can be demonstrated in a way that is assessed achievement pace for this medication repurposing goes from $31 \%$ to $76 \%$. Now for most elevated achievement ratio happens only when utilization of this medication extended in a similar restorative zone of its first sign .To quicken and expand the size of such revelations, a few computational strategies had been proposed for helping in medication reaiming [6]. Calculational medication reaiming strategies could be ordered into target specific, information specific, signature-specific, path or organization specific and focused on instrument specific techniques. This technique centered around various directions characterized by accessible data and explained systems, for example, drugarranged, infection situated and treatmentarranged .These computational medication repositioning techniques empower analysts to inspect practically all medication competitors and test them on a moderately enormous number of sicknesses inside altogether abbreviated timetables. Consequently, mix of translational bioinformatics assets can empower the fast use of medication repositioning on an undeniably more extensive scale .Effective apparatuses are currently accessible for efficient medication repositioning techniques utilizing huge stores of mixes with natural exercises. Coronavirus has arisen by a new Covid, presently known as serious severe respiratory condition
'SARS-CoV-2' [7]. The confirmations on the system of disease, likewise got from past investigations on Covid, propose that an important cycle was the collaboration of virus with spike protein in association with human angiotensin-changing over catalyst 2 'ACE2' and the transmembrane serine protease 2 (TMPRSS2): the receptor with restricting space of protein spike ties to the peptide compound area of humans 'ACE2'.Thus, now the associated last protein expects job of receptor in the authority of infection to the host cell . Job of 'TMPRSS' is identified with this disease cycle, as it is one of host protease enzyme which cut the protein spikes in explicit locales, in this manner actuating the virus passage in the host cell. Fundamental protease enzyme (Mpro) of "SARS-CoV-2" is an important compound, that can assume an essential part in interceding virus replicating and with record. The other virus related proteins agree for infection replicating and then spreading. A silico and a test examination of the communication network among humans and "SARS-CoV2"protein had been as of late distributed and could recommend the main focuses for creating restorative methodologies against this infection. Medication repurposing has just been proposed for explicit medications for treating the current COVID episode .Specifically, a noteworthy number of medications rethought for COVID-19 treatment are or have been utilized in malignant growth treatment .Without a doubt, conceivably appropriate medications acting against the COVID infection are basically influencing transduction of signals, amalgamation of large molecules as well as bioenergetics paths and to those ready for meddling against the host safe reaction, specifically, the dangerous cytokine storm related with serious COVID-19. Furthermore, antiviral mixes are at times viable in battling disease 


\section{DISCUSSION:}

\section{Medication repurposing techniques for COVID-19}

The medication repurposing work process is coordinated uniquely in contrast to conventional medication improvement. In medication repurposing there are less advances and various boundaries following : compound ID, compounding and securing, improvement along with "Food and Drug Administration" (FDA) after wellbeing check. There are a few papers that pre-owned half breed approach , and were arranged, as an instance, the techniques that consisted of both organization as well as bunching together as organization put in the event for us to imagine that organization demonstrating is common over AI. Along these lines, we find just a couple of techniques in which AI models are the predominant system.

\section{Audit of Selected Repurposed Drugs}

Chloroquine and Hydroxychloroquine Hydroxychloroquine and chloroquine both seem as hindering virus passage into cell of humans by restraining glycosylation of human receptor, protein destruction preparation, along with endosomal fermentation. This specialists additionally had immunomodulatory impacts through weakening the cytokines creation along with hindrance of lysosomal and autophagy action on host cell. Dosing of anti-malarial drug to treat Coronavirus has comprised of $500 \mathrm{mg}$ orally on more than one occasion daily.11,12 However, a scarcity of information exists with respect to the ideal portion to guarantee the security and viability of chloroquine. Hydroxychloroquine dosing suggestions for SLE for the most part are $450 \mathrm{mg}$ orally daily.18 Although, a physiologically based pharmacological demonstration study suggest everything that the ideal dosing routine for hydroxychloroquine in COVID treatment is a stacking portion of $450 \mathrm{mg}$ BD by day for one day followed by $250 \mathrm{mg}$ twice daily. Fifteen days conversely, elective proposals are made for $650 \mathrm{mg}$ absolute every day portion dependent on wellbeing's and clinic experience for Whipple based disease. 11 Further investigations are expected to portray the ideal portion for COVID-19.

\section{Lopinavir/ritonavir}

Lopinavir is additionally an inhibitor of the severe respiratory disorder Covid "SARS-CoV" fundamental protease, which is basic for replication and gives off an impression of being profoundly saved in SARS-CoV-2. Atom based unique recreations examination of lopinavir and ritonavir as inhibitors of SARS-CoV 3CL(pro). The most ordinarily used and studied lopinavir/ritonavir dosing regime for COVID-19 treatment is four hundred $\mathrm{mg} / 100 \mathrm{mg}$ doubly daily for up to fourteen days.12,23 Given the so many drug-drug interactions and potential drug reactions which are adverse (summarized in Table 1), sincerest review of concoctions medications and observations area unit needed if these drugs are employed. Adverse effects of ritonavir or loping or embody gastrointestinal distress like nausea and looseness of the bowels (up to $30 \%)$ and hepatotoxicity (4-8\%).24 In patients with coronavirus, these adverse effects is also exacerbated by combination medical aid or infection as a result of close to two hundredth to half-hour of patients have elevated transaminase enzyme based at presentation with coronavirus .

Tribavirin

Tribavirin is guanine that restrains virus RNA-subordinate Ribonucleic acid polymerases. Its action for other $\mathrm{nCoVs}$ can make this antiviral a contender for Coronavirus treatment. Nonetheless, its movement invitro against the virus was restricted and there was requirement of high fixations to restrict virus replication, requiring higher portion and blend treatment. 


\section{Remdesivir}

As of now, it is a promising likely treatment for Coronavirus because of its vast action and range, strong in vitro action against a few nCoVs, incorporating "SARS-CoV-2" with EC90 and EC50 estimations of $0.78 \mu \mathrm{M}$ and $1.75 \mu \mathrm{M}$, respectively.32,5.The security and pharmacokinetics of remdesivir were assessed in single-and various portion stage 1 clinical trials.64 Intravenous imbuements between $4 \mathrm{mg}$ and $226 \mathrm{mg}$ were very much endured with no proof of kidney poisonousness or liver disease . Remdesivir showed direct pharmacokinetics inside this portion range and an intracellular half-existence of more prominent than 35 hours. Following so many portion organizations, reversible aspartate amino transferase based and alanine transaminase based heights had happened. The current portion under scrutiny is a solitary 200-mg stacking portion, trailed by $100-m g$ every day imbuement. No hepatic or kidney changes are suggested as of now, yet inception isn't suggested in patients with an expected glomerular filtration rate under 30 $\mathrm{mL} / \mathrm{min}$. The main clinical utilization of remdesivir was for the treatment of Ebola64; nonetheless, fruitful case reports portraying the utilization of remdesivir for COVID-19 have been reported.65,66 Clinical preliminaries are continuous to assess the wellbeing and antiviral action of remdesivir in patients with gentle to direct or extreme COVID-19.

Different Antivirals

Oseltamivir, a neuraminidase inhibitor endorsed for the treatment of flu, has no recorded in vitro movement against SARSCoV-2.

Umifenovir (otherwise called Arbidol) is an all the more encouraging repurposed antiviral specialist with a novel component of activity focusing on the S protein/ACE2 connection and repressing layer combination of the viral envelope.
Corticosteroids

Dexamethasone (6 $\mathrm{mg}$ for every day) will in general expand thickening component and fibrinogen fixations. Along these lines, it is conceivable for exogenous glucocorticoids to hasten clinical thrombosis.5 furthermore, extended corticosteroid treatment may add to the supposed long COVID disorder that shows with weariness and mental indications, in which steroid-related unfriendly medication responses, for example, myopathy, neuromuscular shortcoming, and mental side effects may have a section to play.

Anticytokine or Immunomodulatory Agents

Monoclonal antibodies coordinated against key incendiary cytokines or different parts of the natural resistant reaction speak to another likely class of adjunctive treatments for COVID-19. The reasoning for their utilization is that the hidden pathophysiology of critical organ harm in the lungs and different organs is brought about by an enhanced safe reaction and cytokine delivery, or "cytokine storm."79 IL-6 has all the earmarks of being a vital driver of this dysregulated irritation dependent on early case arrangement from China. 80 Thus, monoclonal antibodies against IL-6 could hypothetically hose this cycle and improve clinical results. Tocilizumab, a monoclonal neutralizer IL6 receptor enemy, is FDA affirmed to treat RA and cytokine discharge condition following fanciful antigen receptor $\mathrm{T}$-cell treatment.

Immunoglobulin medical care Another potential connected medical care for COVID-19 is that the use of convalescent plasma or hyperimmune immunoglobulins.82 The principle for this treatment is that antibodies from recovered patients might facilitate with each free virus and infected cell immune clearance. 
Anecdotal reports or protocols for convalescent plasma are according as salvage medical care in severe acute respiratory syndrome and MERS.83,84 A 2009 prospective data-based study in ninety three critically unwell patients with H1N1 respiratory illness A, twenty of whom received convalescent plasma, incontestable that receipt of convalescent plasma vs nonreceipt was related to a discount in mortality.

Different Agents

Interferon- $\alpha$ and $-\beta$ are scan for $n C o V s$, with interferon- $\beta$ showing movement against MERS.37,38 Most distributed investigations elaborated consequences of treatment joined with antiviral and to boot lopinavir/ritonavir. Like completely different specialists, postponed treatment might limit viability of those specialists. Given incompatible in vitro and creature data and also the nonattendance of clinical preliminaries, the employment of interferons to treat SARS-CoV-2 cannot presently be suggested.52 Current Chinese rules list interferons as associate degree possibility for mix medical aid.12 many different immunomodulatory specialists usually used for nontransmissible signs exhibit in vitro action or have elements suspected to restrain SARS-CoV-2, including, however not restricted to, baricitinib, imatinib, dasatinib, and cyclosporine. Nitazoxanide, generally an antihelminthic specialist, has wide antiviral action and a moderately great security profile. Nitazoxanide has shown in vitro antiviral movement against MERS and SARS-CoV-2.58,59 Pending additional proof, the antiviral action, immunomodulatory impacts, and security profile of nitazoxanide warrant its further examination as a treatment choice for SARS-CoV-2. Camostatmesylate, an endorsed specialist in Japan for the treatment of pancreatitis, forestalls $\mathrm{nCoV}$ cell passage in vitro through hindrance of the host serine protease, TMPRSS2.3 This tale system gives an extra medication focus to future exploration.

Organization based methodology

Organization based methodologies are crucial and broadly utilized in medication repositioning because of the related capacity to incorporate various information sources [8]. Within the advances of the high-output innovation and genetics techniques, sub-atomic collaborations in the natural frameworks could be demonstrated by the networks .Within these models, the network hubs speak to medications, infections or quality items, while edges speak to corporations or connections between hubs. The subsequent example may encourage the cycle of structure-guided drug and demonstrative examination with the possibility of distinguishing likely new natural targets. Past examinations have proposed that the drug-target organization, the drug-drug organization, medication illness organizations and the protein connection that networks which are helpful within the distinguishing proof of the latest open doors for the drug disclosure or the repositioning .Organization dependent on philosophy joins the framework pharmaceutical based organization medication stage which measures transaction in between infection have drug focuses within the humanised protein-protein based association networks - Organization based bunching approach was stated to find the new medication illness or either medication targeted connections [9].All of this methodologies mean to discover a few modules (drugillness, drug-medication or medication target) utilizing bunching calculations as indicated by the geography structures of organizations. Organization based spread methodologies are another significant sort of organization based methodology. Human Covid (HCoV)- related protein of host gathered from writing and were pooled for creating container protein 
subnetwork of HCoVs. Organization closeness between the drug targets with the $\mathrm{HCoV}$-related proteins were determined in screening concurrent reusable medications for the $\mathrm{HCoVs}$ under the humanised protein interactome models. With utilizing an organizational base strategy, in conceiving for investigating few significant examples valuable for explaining protein which is practically connected with the $\mathrm{HCoVs}$, that are restricted inside this exhaustive humanised interactome model. Besides, they will demonstrate the protein which will fill in according to medication focuses to the particular sickness as they are reasonable medication focuses for potentially antivirals contamination inferable from shared the protein-protein corporations explained by the humanised interactome .First six methodologies [10] dependent on bunching strategies applicable to the protein-protein associations (PPIs), the drug-proteininfection, the drug-target-illness and the medication sickness, while the foremost two methodologies depend on the proliferation philosophy. The two approaches have been clarified inside the content. Besides, these techniques not just give an occasion to improve the presentation of existing strategies yet in addition offer an apparatus to plan more effective and stable methodologies.

\section{Structure-based methodologies}

Virtual screening can help in recognizing little synthetic mixes ready to tie macromolecular focuses within the known or anticipated tertiary structure. It permits screening a huge number of the mix within a restricted period, decreasing expenses in discovering the reasonable in growing newer medications, so as to discover latest focuses for already present medications. The methodology depends mostly on subatomic docking, a computational procedure previously created to see how a substance compound can interface with a natural partner, however these days to a great extent utilized for some different assignments, including drug repurposing [11]. Specialists applied similarity displaying methods to anticipate the structures of some objective microorganism proteins, as an example, the 3-chymotrypsin-like (3CL) proteinase, otherwise called as basic proteinase (Mpro), Spikes, Ribonucleic acid subordinate RNA enzyme (RdRp), helicases and the papain-like (PL) proteinases, that have been distinguished because of main focuses on the antivirals. Such models are used in performing virtual screening of surrounding libraries, along with endorsed medications for centers and the common mixes. Nonetheless, not long former to the seclusion of the COVID-19 virus molecule and gene sequence, within the underlying science network began a remarkable tremendous exertion to tackle the structures of the main proteins associated with viral disease, the replications and dispersal. The Protein Data Bank (PDB), with overall information base on macromolecule structure [12] unlatched a segment devoted to the COVID-19 sections, with its first composition, stored on fifth of February 2020, has been one of SARS-CoV-2 Mpro within complex with the inhibitor recognized through PC supported medication configuration, settled at $2.16 \AA$ goal . The overall convention which was applied was broadly virtually screened of the information bases of the medications, done by various of the docking draws near, regularly following additional calculational conventions, for example, subatomic elements reproductions along with the forecast of the available energy which relates to communication of top hit within the chosen targeted protein, within the end goal for building unwaveringly the quality of the outcome[13]. A potential explanation could be the variety of the chosen beginning information bases and 
the calculations utilized in the docking along with virtual screening. A few examinations were directed focusing on this protein [14].

\section{Artificial intelligence based methodologies}

Man-made intelligence specialists are extremely dynamic to battle COVID-19 impacts, however a couple of documents are giving drug repurposing. Furthermore, albeit few of them have composed within openly accessible, they discovered just a couple of papers acknowledged for distribution and online accessible on a diary, likewise whenever exposed to a quickened peer-survey measure. The framework was coordinated within a criticism from the antiviral exercises from a structure based FIP infection to replicate the test and the implanted AI model was set up to look further and again confirmed by the FIP infection duplicate measure. In the [15] creators utilize formerly prepared profound understanding based medication attack collaboration forecast model, called the Molecular Transforming Drug based Interaction (MT-DTI) to recognize financially accessible medications active against virus that might disturb the viral parts of COVID-19, for example, the proteinases, RNA-subordinate ribonucleic acid polymerases as well as helicases.

Studies on therapeutic options for Covid were reported by Recheet. al. [16] and Thakareet. al [17]. Darwateet. al and Deogade et. al .reported Ayurvedic remedies[18,19]. Jameel et. al reported on drugs and therapies for children [20]. Joseph et. al. described on palliative care of Covid patients [21]. Afaque reported about treatment of diabetes in patients affected by covid-19[22]. Some interesting studies were reported by Agrawal et. al [23], Dhole et. al.[24] and Gulhane et. al. [25].

\section{CONCLUSION :}

Medication replacing is the field of medication study whose significance was expanding from the previous decades, because of a few favorable circumstances, for example, the likelihood to abbreviate the subjective preliminaries, with the expansion of life of the former medication by the finding another restorative objective and the revelation of frequently obscure connections among obviously removed infections. The direness to discover medications to confront COVID19 outbreak massively pushed back this sort of examination into the previous time. Calculational methodologies have played and yet assume a significant job to look through weapons powerful against SARSCoV-2 infection within the arms stockpile of medications accessible today, however to date, the outcomes don't seem to satisfy hopes. Based on the writing, a significant job in this test of skill and endurance has been played by underlying genomics, to whose commitment has been made conceivable additionally through the uncommon rate with which the form of the main viral proteins were made accessible. Nonetheless, numerous consequences of these investigations seem not completely persuading. Not many examinations on a similar objective join on similar medications, not very many give a verifiable proof of an impact and practically none gives a trial approval. Besides, numerous examinations foresee the adequacy of talked about medications that neglected to exhibit their viability in clinical preliminaries.

\section{REFERENCES :}

[1] Jin G, Wong ST. Toward better medication repositioning: organizing and incorporating existing techniques into proficient pipelines. Medication Discov Today 2014; 19:637-44. 
[2] Talevi A, Bellera CL. Difficulties and openings with drug repurposing: discovering techniques to discover elective employments of therapeutics. Master Opin Drug Discov 2020; 15:397-401.

[3] de Oliveira EA, Karen LL. Medication repositioning: idea, arrangement, approach, and significance in uncommon/vagrants and dismissed infections. J Appl Pharm Sci 2018; 8:157-65.

[4] Hernandez JJ, Pryszlak M, Smith L, et al. Allowing drugs another opportunity: beating administrative and monetary obstacles in repurposing affirmed drugs as disease therapeutics. Front Oncol 2017; 7:273.

[5] Khan RJ, Jha RK, Amera GM, et al. Focusing on SARS-CoV-2: a deliberate medication repurposing way to distinguish promising inhibitors against 3C-like proteinase and 2-O-ribose methyltransferase. J BiomolStructDyn 2020;1-14. Distributed online in front of print, 20 April 2020)

[6] Li J, Zheng S, et al. An overview of latest things in computational medication repositioning. Brief Bioinform 2016; 17:2-12.

[7] Pawar AY. Fighting destroying COVID-19 by drug repurposing. Int J Antimicrob Agents 2020; 56:105984.

[8] Zhou Y, Hou Y, Shen J, et al. Organization based medication repurposing for novel Covid 2019nCoV/SARS-CoV-2. Cell Discov 2020; 6:14.

[9] Barlow A, Landolf KM, Barlow B, et al. Audit of arising pharmacotherapy for the treatment of Covid sickness 2019. Pharmacotherapy 2020; 40:416-37.

[10] Sadegh S, Matschinske J, Blumenthal DB, et al. Investigating the SARS-CoV-2 infection have drug interactome for drug repurposing. Nat Commun 2020; 11: 3518.

[11] Kumar S, Kumar S. Sub-atomic docking: a structure-based methodology for drug repurposing. In: In Silico Drug Design. Repurposing Techniques and Methodologies, 2019. Roy K, ed. pp. 161-189.

[12] Berman HM, Henrick K, Nakamura H. Declaring the overall Protein Data Bank. Nat Struct Biol 2003; 10: 980.

[13] Alonso H, Bliznyuk AA, Gready JE. Consolidating docking and atomic dynamic reproductions in medication plan. Drug Res Rev 2006; 26:53168.

[14] Abo-Zeid Y, Ismail NS, McLean GR, et al. A sub-atomic docking study repurposes FDA affirmed iron oxide nanoparticles to treat and control COVID-19 contamination. Eur J Pharm Sci 2020; 153:105465.

[15] Beck BR, Shin B, Choi Y, et al. Foreseeing industrially accessible antiviral medications that may follow up on the novel Covid (SARS-CoV2) through a medication target association profound learning model. ComputStructBiotechnol J 2020; 18:784, 790.

[16] Reche, A., R. Kolse, S. Gupta, A. Ingle, K.G. Chhabra, and G. Nimbulkar. "Therapeutic Options for COVID - 19: Pandemic - a Review." International Journal of Research in Pharmaceutical Sciences 11, no. Special Issue 1 (2020): 42024.

https://doi.org/10.26452/ijrps.v11iSP L1.2802.

[17] Thakre, P., W.M. Naqvi, T. Deshmukh, N. Ingole, and S. Deshmukh. "Crucial Role of Physiotherapy in Treating Covid-19 Patients." International Journal of Research in Pharmaceutical Sciences 11, no. Special Issue 1 (2020): 96771. 
https://doi.org/10.26452/ijrps.v11iSP L1.3300.

[18] Darwate, T., and S. Hiware. "Concept of Rasayana Therapy in Covid 19." International Journal of Research in Pharmaceutical Sciences 11, no. Special Issue 1 (2020): 85761.

https://doi.org/10.26452/ijrps.v11iSP L1.3093.

[19] Deogade, M.S. "Agnihotra (Homa)an Ayurveda Therapy in the Prevention and Control of Covid19." International Journal of Research in Pharmaceutical Sciences 11, no. Special Issue 1 (2020): 3049.

https://doi.org/10.26452/ijrps.v11iSP L1.2717.

[20] Jameel, P.Z., and A.M. Taksande. "COVID-19: A Review of Drugs and Therapies for Children." Sri Lanka Journal of Child Health 49, no. 3 (2020): 210-17. https://doi.org/10.4038/sljch.v49i3.9 136.

[21] Joseph, M.B., S. Pohekar, A. Raut, and M. Patil. "The Palliative Care and Covid-19 Pandemic." International Journal of Research in Pharmaceutical Sciences 11, no. Special Issue 1 (2020): 618-22. https://doi.org/10.26452/ijrps.v11iSP L1.2861.

[22] Afaque, S.Y. "Association and Treatment of Diabetes in Patients Affected by COVID-19." International Journal of Research in Pharmaceutical Sciences 11, no. Special Issue 1 (2020): 1198-1201. https://doi.org/10.26452/ijrps.v11iSP L1.3591.

[23] Agrawal, A., P. Fulzele, K. Deo, Z.S. Quazi, and A. Daryapurkar. "Engineered Nano-in-Micro Drug Delivery Scheme against Sars-Cov2: A Hypothesis." European Journal of Molecular and Clinical Medicine 7, no. 7 (2020): 1892-1901.
[24] Dhole, P.D., V.K. Lohe, R.P. Kadu, S.C. Mohod, M. Meshram, and G.A. Thakare. "Post COVID-19 Protocol of Treatment, Radiologic Examination and Infection Control in Dentistry." International Journal of Research in Pharmaceutical Sciences 11, no. Special Issue 1 (2020): 138489.

https://doi.org/10.26452/ijrps.v11iSP L1.3664.

[25] Gulhane, A., and S. Hiware. "Nutritional Recommendation for COVID-19." International Journal of Research in Pharmaceutical Sciences 11, no. Special Issue 1 (2020): 75357. https://doi.org/10.26452/ijrps.v11iSP L1.3078. 